\title{
Involvement of Inferior Parietal Lobules in Prospective Memory Impairment during Acute MDMA (Ecstasy) Intoxication: An Event-Related fMRI Study
}

\author{
Johannes G Ramaekers*,', Kim PC Kuypers', Marleen Wingen', Armin Heinecke² and Elia Formisano² \\ 'Department of Neuropsychology and Psychopharmacology, Faculty of Psychology and Neuroscience, Maastricht University, Maastricht, The \\ Netherlands; ${ }^{2}$ Department Cognitive Neuroscience, Faculty of Psychology and Neuroscience, Maastricht University, Maastricht, The Netherlands
}

\begin{abstract}
Prospective memory refers to the realization of delayed intentions. Several studies have shown that 3,4-methylenedioxymethamphetamine (MDMA) users perform worse on measures of prospective memory as compared to nondrug users. Interpretation of these data may be limited because of polydrug use, psychosocial stressors, and increased psychopathology that have been reported in MDMA users. This study was designed to directly assess the pharmacological effect of MDMA on prospective memory and brain activity in a double-blind, placebo-controlled, cross-over study. Twelve recreational MDMA users received MDMA 75 mg and placebo and performed an objective prospective memory task during functional imaging. During prospective memory task performance subjects were engaged in a foreground task that consisted of a simple reaction time to visual stimuli (Go trials) and a prospective task of withholding a response during trials that were part of a dynamic memory set (No go trials). Behavioral data showed that a single dose of MDMA increased prospective memory failures in the No go trials, and that number of prospective memory failures was positively correlated to MDMA concentration in plasma. Functional imaging showed that MDMA decreased BOLD activation during Go trials in the thalamus (left), putamen (left), precuneus (left), and the inferior parietal lobules (bilateral), as compared to placebo. During No go trials, MDMA reduced BOLD deactivation in the inferior parietal lobules (bilateral), as compared to placebo. It is concluded that the loss of deactivation in inferior parietal lobules may account for increments in memory failures observed during MDMA intoxication.

Neuropsychopharmacology (2009) 34, I64I-1648; doi: I0.1038/npp.2008.219; published online 17 December 2008
\end{abstract}

Keywords: MDMA; prospective memory; acute; imaging

\section{INTRODUCTION}

Users of 3,4-methylenedioxy-methamphetamine (MDMA) have repeatedly been associated with learning and verbal memory deficits on a range of neuropsychological tests (Cole and Sumnall, 2003; Gouzoulis-Mayfrank et al, 2002; Morgan, 2000; Parrott, 2000, 2001; Zakzanis et al, 2007). The prototypical example being a reduction in performance of abstinent MDMA users on immediate and delayed word recall tasks as compared to a group of controls (non-drug users). Verbal memory deficits appear most evident in heavy MDMA users who have been abstinent for over 6 months. The cause of these persistent memory deficits has been a matter of continuous debate because a number of confounders have hampered the interpretation of these

\footnotetext{
*Correspondence: DrJG Ramaekers, Department of Neuropsychology and Psychopharmacology, Faculty of Psychology and Neuroscience, Maastricht University, PO Box 616, Maastricht, The Netherlands, Tel: + 3143388 1951, Fax: + 31433884125 ,

E-mail: j.ramaekers@psychology.unimaas.nl

Received 15 September 2008; revised 19 November 2008; accepted 19 November 2008
}

data. Most notably, MDMA users are usually polydrug users, which makes it difficult to attribute their memory impairments to a single drug (Cole and Sumnall, 2003; Gouzoulis-Mayfrank and Daumann, 2006). Consequently, memory deficits in MDMA users have been attributed to MDMA (Bolla et al, 1998; Halpern et al, 2004; Morgan, 1999; Thomasius et al, 2006; Verkes et al, 2001) or other drugs such as cannabis (Croft et al, 2001; Dafters et al, 2004; Montgomery et al, 2005; Simon and Mattick, 2002) or amphetamines (Jager et al, 2008).

Generally, memory deficits reported in current or abstinent MDMA users appear relatively mild (Bedi and Redman, 2008a; Zakzanis et al, 2007). Yet, even mild drug effects may interfere with everyday aspects of memory, such as prospective memory. The latter involves remembering of future intentions such as remembering to call a friend, take medication, or go to a meeting, and differs from retrospective memory (recalling past events or knowledge) in that it involves self-initiated retrieval, sometimes cued by an event or time (Einstein et al, 2005; McDaniel and Einstein, 1993). MDMA users have reported more prospective memory errors than controls as assessed by 
prospective memory questionnaires (Heffernan et al, 2001a, b; Montgomery and Fisk, 2007) and performed worse than controls on objective laboratory measures of prospective memory (Rendell et al, 2007; Zakzanis et al, 2003). As can be expected, some studies have also concluded that prospective memory impairments reported by MDMA users are related to concurrent cannabis use (Rodgers et al, 2001, 2003). Others have noted that subjective self-reports may not be the best tools for studying prospective memory in MDMA users because perceived memory disturbances might be confounded by psychosocial or lifestyle stressors such as sleep deprivation or increased psychopathology that have been reported in MDMA users as well (Bedi and Redman, 2008b; Hanson and Luciana, 2004; Kuypers et al, 2008; Lieb et al, 2002; McGuire, 2000; Rendell et al, 2007). These studies therefore provide limited information regarding the extent and practical implications of MDMA induced memory impairments in everyday life.

Only few researchers have attempted to directly assess the pharmacological effect of MDMA on human memory performance in placebo-controlled studies. Kuypers and Ramaekers (2005, 2007) administered single doses of MDMA $75 \mathrm{mg}$, methylphenidate $20 \mathrm{mg}$, and placebo to 18 recreational users. They demonstrated that performance on a word learning and spatial memory task was impaired by MDMA at $1.5 \mathrm{~h}$ after drug administration when blood concentration were maximal but returned to placebo levels after $24 \mathrm{~h}$ (withdrawal phase) when blood concentrations were low. In addition, these researchers were able to conclude that MDMA induced memory impairment was related to the drug's serotonergic but not dopaminergic mechanism of action, because methylphenidate, a dopaminergic stimulant, did not affect memory performance at all. The same research group (Kuypers et al, 2008) also demonstrated that MDMA induced impairments of verbal and spatial memory were aggravated by sleep loss during a night of sleep deprivation. Hasler et al (2008) demonstrated that a single dose of MDMA $(1.6 \mathrm{mg} / \mathrm{kg})$ impaired associative learning and working memory at $2 \mathrm{~h}$ after administration, relative to placebo. Acute studies have thus confirmed the potential of MDMA to (transiently) impair memory and may serve as valuable, well-controlled models to further define neuropharmacological mechanisms and neural networks underlying MDMA induced memory impairment.

This study was designed to assess prospective memory function of MDMA users after a single dose of MDMA in a double-blind, placebo-controlled, cross-over study. A computer-based prospective memory task was developed in the laboratory. Conceptually it consisted of an instruction to subjects to remember to withhold a response to specific future events (event-based) while they were otherwise engaged in a different task. In addition, the prospective memory task was designed to also enable eventrelated fMRI measurements during task performance after drug administration. Previous neuroimaging studies have shown a consistent pattern of activation in the anterior prefrontal cortex, the inferior parietal regions, the precuneus, and the hypothalamus during performance of prospective memory tasks (Burgess et al, 2001, 2003; den Ouden et al, 2005). It was expected that MDMA effects would be associated with changes in brain activation in any of these regions. This study was designed to provide (1) direct evidence for the association between MDMA and memory impairment; (2) objective assessments of prospective memory function during MDMA intoxication; and (3) neural structures critically involved in memory dysfunction during MDMA intoxication.

\section{METHODS}

\section{Subjects, Design, and Treatments}

The study followed a double-blind, placebo-controlled, twoway crossover design. Complete balancing of the treatment orders yielded two treatment orders randomly assigned to subjects. Twelve recreational users of ecstasy were included in the study. Two subjects were unable to conduct the prospective memory task according to protocol and were excluded and replaced. In one case, the subject conducted the foreground task but not the prospective task (for explanations see Methods section) and in another case, the subject stopped responding to all tasks after $10 \mathrm{~min}$. Twelve recreational users (nine men and three women) produced complete behavioral and imaging data sets. Their age ranged between 19 and 29 years (mean 23.3 years). Lifetime use of ecstasy varied between subjects ranging from light ( $<15$ occasions, five subjects), to moderate (16-40 occasions, six subjects), and heavy use ( $>500$ occasions, one subject). Mean (SE) lifetime use of ecstasy was 60 occasions (139.2). Recent drug surveys in the Netherlands have shown that ecstasy pills on the Dutch market on average contain $80 \%$ MDMA (Trimbos, 2007). Lifetime use of alcohol, cannabis, and amphetamines varied in a similar way between subjects.

Initial screening was accomplished on the basis of a questionnaire on medical history. Subjects who were accepted were examined by the medical supervisor, who also checked vitals signs and took blood and urine samples. Standard blood chemistry, hematological, and drug screen tests were conducted on these samples. Inclusion criteria were: experience with the use of MDMA; free from psychotropic medication; good physical health as determined by examination and laboratory analysis; absence of any major medical, endocrine, and neurological condition; normal weight, body mass index (weight/height ${ }^{2}$ ) between 18 and $28 \mathrm{~kg} / \mathrm{m}^{2}$; and written informed consent. Exclusion criteria were: history of drug abuse (other than the use of MDMA) or addiction; pregnancy or lactation; cardiovascular abnormalities as assessed by standard 12-lead ECG; excessive drinking ( $>20$ standard alcoholic consumptions a week); hypertension (diastolic $>100$, systolic $>170$ ); history of psychiatric or neurological disorder; and presence of objects in the body with magnetic properties.

The study was conducted according to the code of ethics on human experimentation established by the Declaration of Helsinki Principles (1964) and amended in Edinburgh (2000). Approval for the study was obtained from the University's Medical Ethics Committee. A permit for obtaining, storing, and administering MDMA was obtained from the Dutch drug enforcement administration. All subjects entered a double-blind, placebo-controlled, randomized, crossover study design with balancing of treatments. 
The prospective memory task was conducted at $2 \mathrm{~h}$ postdrug administration. The treatments were single doses of MDMA $75 \mathrm{mg}$ and placebo on separate treatment days. Wash out period between treatments was at least 7 days. MDMA and placebo were administered orally in identically appearing formulations. MDMA was administered as a $25 \mathrm{ml}$ solution in bitter orange peel syrup, which was ingested at once with $200 \mathrm{ml}$ of orange juice.

\section{Procedures}

Subjects were asked to refrain from any drugs during study participation starting 1 week before the medical screening and physical examination. The subjects were not allowed to use alcohol on the day before an experimental session and were requested to arrive at experimental sessions well rested. Drug and alcohol screens in breath and urine were performed before experimental sessions upon arrival of the subject. MDMA and placebo were only administered in case a subject had passed the urine drug screen on a given test day. All subjects received a training session before onset of the experimental sessions to familiarize them with the tests and procedures.

\section{Prospective Memory Task}

This event-based prospective memory task was developed to assess the subjects' ability to remember and execute future plans. Conceptually, such tasks can be created in the laboratory by instructing subjects to make a special response to a specific future event while they are otherwise engaged in a different task. In this study subjects were engaged in a foreground task that consisted of a simple reaction time to stimuli presented on a screen. Subjects were also given a prospective task, ie, to withhold a response during trials that were part of a dynamic memory set.

The foreground task consisted of 100 successive presentations of a letter (A or B) in the center of a computer screen. Subjects were required to respond to each letter as quickly as possible by pressing one of two response buttons. One button was pressed to indicate that the letter ' $A$ ' appeared and the other to indicate the letter ' $B$ '. Both letters were presented equally often. Subjects were informed about the trial number by means of a trial counter that was always present in the left top corner of the screen. In addition, subjects were presented at irregular times with a future trail number in the right top corner of the display. Subjects were instructed to remember this future trial number and withhold from responding to the foreground task during the actual occurrence of the future trial. The memory set of subjects was dynamic and contained up to three future trial numbers. A trial number in the memory set would be replaced by a novel future trial number whenever the actual trial number matched a future trail number in the set. Trials during which subjects were expected to respond were classified as Go trials. Trials during which subjects were instructed to withhold a response were classified as No go trials (prospective memory trials). Time between presentation of a future trial number and the actual occurrence of the trial (ie, memory delay) varied between 1,2 , and 3 min, equally divided over all No go trials. Each trial lasted for $12 \mathrm{~s}$. However, the central letters disappeared at any given button press. Presentations of future trial numbers lasted $4 \mathrm{~s}$. In total, the prospective memory task consisted of 68 Go trials and 24 No go trials. At the beginning and the end of the task, a total of eight trials were presented during which the memory set was empty. Number of prospective memory failures in No go trials was the primary dependent performance parameter. Number of correct responses and reaction time during Go trials were the secondary dependent performance parameters. The prospective memory task lasted for $20 \mathrm{~min}$ and was administered to subjects during functional imaging in a $3 \mathrm{~T}$ MR scanner. Two parallel versions of the prospective memory task were developed for administration during the two treatment periods (placebo and MDMA) to avoid learning effects.

\section{fMRI Data Acquisition and Data Processing}

Measurements were acquired using a $3 \mathrm{~T}$ Siemens Allegra MR scanner. A T1-weighted anatomical scan was acquired for each participant using a 3D modified driven equilibrium Fourier transform sequence (176 slices; in-plane resolution, $1 \mathrm{~mm}^{2}$ ). A $\mathrm{T} 2 *$-weighted functional measurement was acquired using an echo-planar image pulse sequence (1316 whole-brain volumes; 32 slices; slice thickness, $3.5 \mathrm{~mm}$; no slice gap; flip angle $90^{\circ}$; TR/TE, $2000 / 30 \mathrm{~ms}$; in-plane resolution, $3.5 \times 3.5 \mathrm{~mm}^{2}$, matrix size, $\left.64 \times 64\right)$ and interleaved slice sampling. Functional time series consisted of 600 volumes, and lasted $20 \mathrm{~min}$. Data processing and analysis were conducted using Brainvoyager QX, version 1.6 (Brain Innovation, Maastricht, the Netherlands). Preprocessing consisted of 3D motion correction, slice time correction, linear trend removal of time courses, and high pass filtering of three cycles per time course. Individual anatomical data sets were normalized to standard 3D space (Talairach and Tournoux, 1988). Individual functional images were realigned, coregistered, and normalized to the anatomical data, and resampled to a voxel size of $3 \times 3 \times 3 \mathrm{~mm}^{3}$. Functional time series were linked to prospective memory task sequence and performance by means of individual protocol files that identified the timing of Go and No go trials.

\section{Statistical Analysis}

Behavioral data of the prospective memory task were analyzed by means of a GLM repeated measures ANOVA (SPSS 15.0) with MDMA (two levels, ie, MDMA $75 \mathrm{mg}$ and placebo) as the main factor for the analysis of Go trials and MDMA (two levels), and memory delay (three levels; ie, 1, 2, and $3 \mathrm{~min}$ ) as the main factors for the analysis of No go trials. The $\alpha$-criterion significance level was set at $p=0.05$.

Functional imaging data collected during the prospective memory task was analyzed by means of a whole brain, RFX repeated measures ANOVA (Brainvoyager QX 1.6) with MDMA (two levels) as the main factor in Go trials and MDMA (two levels) and memory delay (three levels) as the main factors in No go trials. Statistical analyses were restricted to the initial $6 \mathrm{~s}$ of each trial that generated a correct behavioral response. The $\alpha$-criterion significance level was set at $p=0.001$ with a minimum cluster size of 25 
voxels to account for multiple comparisons (Forman et al, 1995; Poldrack et al, 2008).

\section{Pharmacokinetic Assessments}

MDMA was determined in plasma at $1.25 \mathrm{~h}$ postdrug. Blood samples were centrifuged immediately and subsequently frozen at $-20^{\circ} \mathrm{C}$ until analyses for pharmacokinetic assessments. MDMA and MDA concentrations were determined using solid phase extraction and gas chromatography with mass spectrometric detection with quantification limits of $2.5 \mathrm{ng} / \mathrm{ml}$.

\section{RESULTS}

\section{Pharmacokinetics}

Mean (SE) MDMA concentration at $1.25 \mathrm{~h}$ after MDMA administration was $79.1(23.1) \mathrm{ng} / \mathrm{ml}$. MDMA concentrations varied between individuals and ranged between 36.4 and $113.4 \mathrm{ng} / \mathrm{ml}$. MDA concentrations were below the limit of quantification in 9 out of 12 subjects. In three subjects MDA concentrations ranged between 2.5 and $3.7 \mathrm{ng} / \mathrm{ml}$.

\section{Behavioral Data}

ANOVA revealed a significant main effect of MDMA on the number of prospective memory failures in the No go trials $\left(\mathrm{F}_{1,11}=9.13 ; p=0.012\right)$. The average number of prospective memory failures were $1.0(\mathrm{SE}=0.25,95 \% \mathrm{CI}: 0.46-1.5)$ during placebo and 2.75 ( $\mathrm{SE}=0.57 ; 95 \% \mathrm{CI}$ : $1.5-4.0)$ after MDMA. Prospective memory failures thus increased during MDMA intoxication, relative to placebo. The effect size and observed power of the observed difference in memory failures were 0.45 and 0.79 , respectively. Prospective memory failures were positively correlated to MDMA concentration $(r=0.63 ; p=0.028)$. Lifetime use of ecstasy was not significantly correlated to MDMA induced prospective memory failures. A summary of mean prospective memory failures (averaged over memory delay) during MDMA and placebo treatment is given in the left panel of Figure 1. Correlation of MDMA concentration and prospective memory failure is shown by means of a linear regression equation in the right panel of Figure 1. The factors memory delay and MDMA $\times$ memory delay did not affect prospective memory failure. Number of correct responses and reaction time during Go trials were not affected by any factor. Mean (SE) RT during MDMA and placebo treatments were 1853 (166) and 1974 (180) ms, respectively.

\section{Functional Imaging Data}

RFX repeated measures ANOVA identified significant main effects of MDMA in five brain areas $\left(\mathrm{F}_{1,11}>19.69 ; p<0.001\right)$ during performance in the Go trials. These areas included the thalamus (left), putamen (left), precuneus (left), the inferior/superior parietal lobule (left), and the inferior parietal lobule (right). The coordinates of these clusters and their voxel sizes are summarized in Table 1. Brain activations in each of these areas and their event-related averages of BOLD responses during MDMA and placebo are depicted in Figure 2. They show that MDMA reduced BOLD activation as compared to placebo.

RFX repeated measures ANOVA also identified significant main effects of MDMA in two brain areas $\left(F_{1,11}>19.69\right.$; $p<0.001)$ during performance in the No go trials. The areas

Table I Summary of Talairach Coordinates and Cluster Size of Anatomical Regions Showing a Significant Main Effect of MDMA in Go Trials and No Go Trials

\begin{tabular}{lcrrrr}
\hline Brain area & BA & Voxels & $X$ & $Y$ & $Z$ \\
\hline Go trials & & & & & \\
$\quad$ Thalamus (left) & & 62 & -8 & -7 & 4 \\
$\quad$ Putamen (left) & & $7 \mid$ & -12 & 6 & 6 \\
$\quad$ Precuneus (left) & 7 & $7 \mid$ & -21 & -67 & 4 | \\
Inferior/superior parietal lobule (left) & $40 / 7$ & 63 & -33 & -48 & 50 \\
$\quad$ Inferior parietal lobule (right) & 40 & 142 & 56 & -30 & 34 \\
& & & & & \\
No go trials & & & & & \\
$\quad$ Inferior parietal lobule (left) & 40 & 32 & -39 & -46 & 44 \\
Inferior parietal lobule (right) & 40 & 31 & 33 & -55 & 43 \\
\hline
\end{tabular}
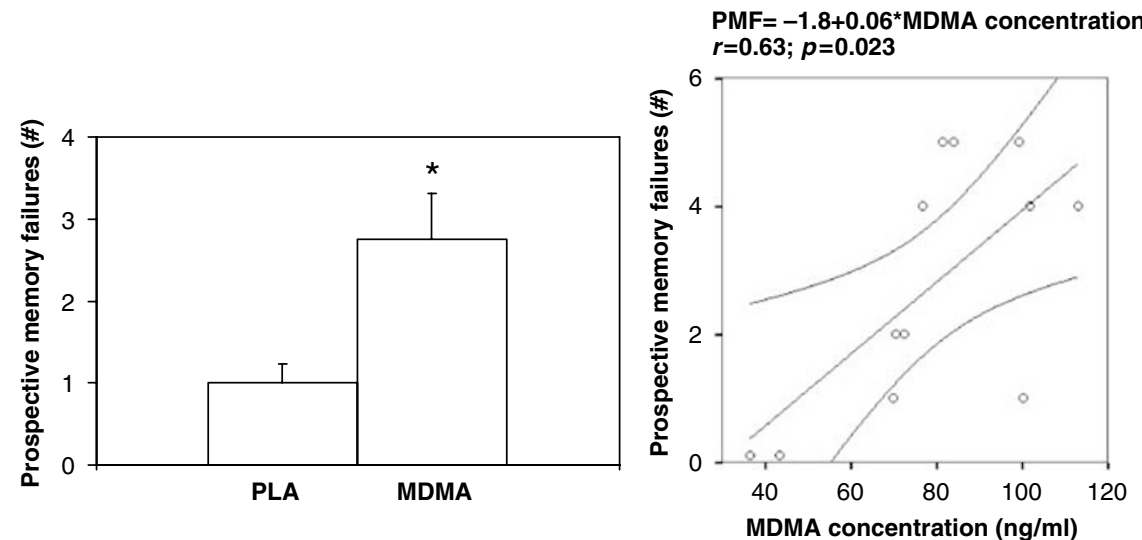

Figure I The left panel shows mean (SE) prospective memory failures after MDMA and placebo administration during No go trials. The right panel shows a linear regression $(95 \% \mathrm{Cl})$ between MDMA concentration and prospective memory failures in 12 subjects $\left(\mathrm{PMF}=\right.$ prospective memory failures; $\left.{ }^{*} p<0.05\right)$. 

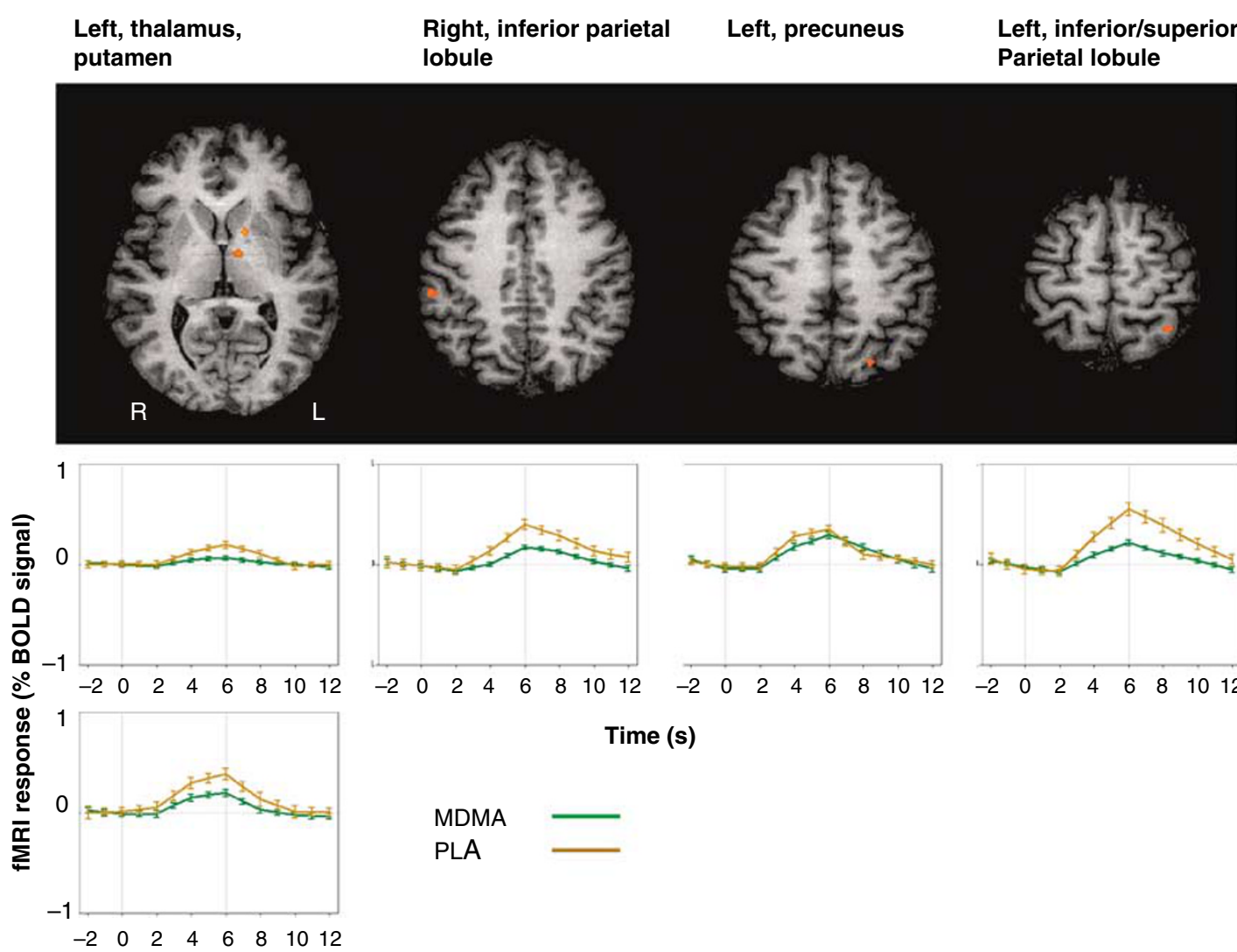

Figure 2 Transversal sections of the brain showing significant MDMA effects during Go trials in five brain areas. Event-related BOLD responses averaged over Go trials during MDMA and placebo are given in the lower panels for each brain area.
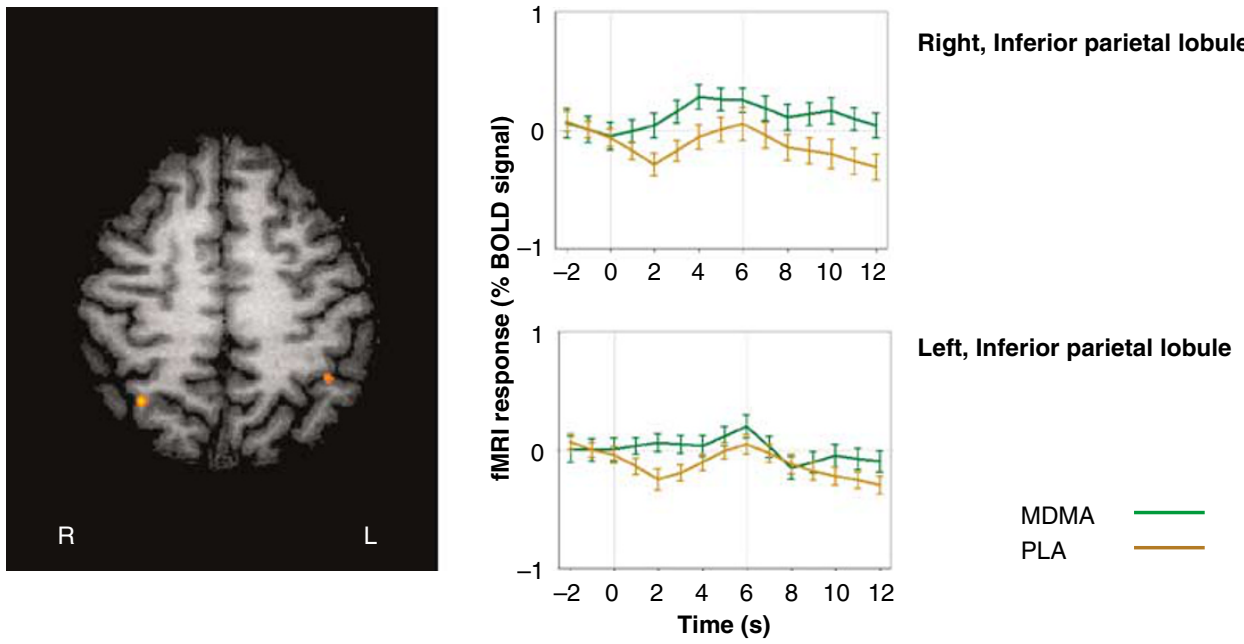

Figure 3 Transversal section of the brain showing significant MDMA effects during No go trials in two brain areas. Event-related BOLD responses averaged over No go trials during MDMA and placebo are given in the right panels for each brain area.

included the inferior parietal lobules in both hemispheres. The coordinates of these clusters and their voxel sizes are summarized in Table 1. Brain activations in each of these areas and the associated event-related averages of BOLD responses during MDMA and placebo are depicted in Figure 3. They show that MDMA reduced BOLD deactivation as compared to placebo. Prospective memory failures were positively correlated to BOLD activation in the left $(r=0.457 ; p=0.025)$ and right $(r=0.46 ; p=0.024)$ inferior parietal lobule.

\section{DISCUSSION}

This study was designed to establish the detrimental effects of MDMA on prospective memory during intoxication in a placebo-controlled study, to develop an objective laboratory 
task for measuring prospective memory, and to determine neuroanatomical structures that underlie MDMA induced memory deficits.

A single dose of MDMA $75 \mathrm{mg}$ increased the number of prospective memory failures during No go trials in the prospective memory task. The detrimental effect of MDMA was also concentration dependent. Subjects with a low MDMA concentration showed less prospective memory failures as compared to subjects with a high MDMA concentration. On average, the magnitude of the MDMA effect on prospective memory was rather mild as indicated by a medium effect size of 0.45 . The detrimental effect of MDMA was not correlated to lifetime use. The latter indicates that acute effects of MDMA on prospective memory occur in MDMA users independent of their lifetime use history in the present subject population. However, it should be noted that heavy users where underrepresented in the present sample. A definitive answer to the question whether heavy use of ecstasy affects the magnitude of acute MDMA effects on memory should come from a direct comparison between acute MDMA effects in light and heavy users.

It could be argued that the present MDMA effect on prospective memory task performance may reflect a loss of motor impulse control during No go trials rather than a loss of prospective memory. That is, the prospective memory task in part is based on a Go/No go paradigm that is traditionally used for measuring motor impulsivity. However, previous work has shown that single doses of MDMA actually increase motor impulse control in Go/No go paradigms (Ramaekers and Kuypers, 2006) suggesting that prospective memory failures did not result from increased levels of motor impulsivity. The notion of MDMA induced impairment of prospective memory is also in line with previous studies demonstrating that single doses of MDMA impair (retrospective) memory during intoxication in placebo-controlled studies (Hasler et al, 2008; Kuypers and Ramaekers, 2007; Kuypers et al, 2008). The present data also confirm earlier reports from retrospective studies showing that MDMA users perform worse on subjective and objective measures of prospective memory as compared to non-drug using controls (Heffernan et al, 2001b; Rendell et al, 2007).

It is noteworthy that a high inter-subject variability in MDMA concentration was found at $1.25 \mathrm{~h}$ past administration. MDMA concentrations after administration of a single dose of $75 \mathrm{mg}$ ranged between 36.4 and $113.4 \mathrm{ng} / \mathrm{ml}$, indicating that MDMA concentration could be approximately threefold higher in some subjects as compared to others. Pharmacokinetic reports of high inter-subject variability after single doses of MDMA have been published before (de la Torre et al, 2000, 2004; Farre et al, 2004; Kolbrich et al, 2008; Mas et al, 1999). It has been postulated that individuals genetically deficient for the hepatic enzyme CYP2D6 may exhibit higher concentrations of MDMA because the enzyme be involved in the $N$-demethylation of MDMA to its metabolite MDA (de la Torre et al, 2004). Yet, it has also been demonstrated that MDMA produces non-linear pharmacokinetics at differential recreational doses in all subjects, independent of CYP2D6 genotype (de la Torre et al, 2004). The pharmacological mechanism underlying high intersubject variability of MDMA concentration is not yet fully understood. The notion of inter-subject variability, however, is very relevant as it implies that subjects who demonstrate a high MDMA concentration after a single dose of MDMA will be more prone to develop acute neurotoxicity and/or behavioral toxicity as evinced by larger prospective memory impairments in this study.

Prospective memory refers to the functions that enable a person to carry out an intended act after a delay. Such goal directed behavior depends on keeping relevant information in mind and irrelevant information out of mind. Previous neuroimaging studies have demonstrated a consistent pattern of activation during performance of prospective memory tasks (Burgess et al, 2001, 2003; den Ouden et al, 2005). The main brain structures included the anterior prefrontal cortex, the inferior parietal regions, the precuneus, and the hypothalamus. Little, however, is known about the roles of these structures in prospective memory. In general, neuroimaging studies demonstrated that each of these structures may be involved in the maintenance of an intention as well the actual realization of the intention. In this study, it was expected that prospective memory task performance would involve similar brain structures as in previous neuroimaging studies and that detrimental effects of MDMA would be associated with brain activation in any of these regions.

Present imaging data demonstrated MDMA effects on brain activity in several of the expected areas. A main effect of MDMA was observed during Go trials in the hypothalamus (left), putamen (left), precuneus (left), and bilaterally in the inferior parietal lobules. Event-related averages of BOLD responses during Go trials showed that BOLD activation during MDMA treatment was always less as compared to placebo in all of these regions. In addition, a main effect of MDMA was found during No go trials in the inferior parietal lobules (bilateral). Event-related averages demonstrated that BOLD responses during No go trials were always deactivated, and that BOLD deactivation was less during MDMA as compared to placebo. In other words, a negative BOLD response (deactivation) was observed during placebo whereas no or a slightly positive BOLD response were observed during MDMA. There was no apparent association between the main effects of MDMA on BOLD response during Go trials and behavioral parameters during Go trials: ie, the number of correct responses and reaction time during Go trials did not differ between MDMA and placebo. During No go trials, however, the suppressing effect of MDMA on BOLD deactivation in the inferior parietal lobules was supported by an increase in prospective memory failures.

The inferior parietal lobules were the only brain structures that demonstrated a main effect of MDMA during Go trials as well as No go trials. The role of the inferior parietal lobules has been investigated previously in a number of neuroimaging studies employing arithmetic tasks. These studies have consistently associated the inferior parietal lobules with quantitative number representations and the ability to perform simple calculations and number comparisons (Gobel et al, 2004; Piazza et al, 2002; Pinel et al, 1999; Sandrini et al, 2004). Other studies have indicated that these brain structures are specifically involved in self-action monitoring (Schnell et al, 2007) 
and the reestablishment of executive control over previously automatized behavior (Kubler et al, 2006). There is a clear similarity between number comparison tasks and the prospective task memory in this study. On top of the foreground task subjects were always actively engaged in remembering prospective trial numbers and comparing them to actual trial numbers presented on the screen. If the actual trial number did not match a prospective trial number then subjects could respond to the foreground task as instructed. However, in case of a match, subjects were told to withhold their response. BOLD activation during Go trials in the inferior parietal lobules thus may reflect a mismatch between actual and prospective trial numbers and the selection of a response. BOLD deactivation during No go trials on the other hand may reflect a match between actual and prospective trail numbers and the inhibition of a response.

The present data suggests that a reduction in BOLD deactivation in the inferior parietal lobules during No go trials after MDMA may underlie the MDMA induced increase in prospective memory failures. It should be noted, however, that analyses of imaging data were restricted to No go trials that elicited a correct behavioral response (ie, withholding a response). As such, the imaging data shown for No go responses actually show correct responding on No go trails. The association between memory failures and reduced BOLD deactivation would be less inferential if it could be based on errors during No go trials. Unfortunately the average number of error trials are too low (ie, 1 and 2.7 out 24 trials during placebo and MDMA, respectively) to allow any meaningful analyses of associated BOLD responses. However, the fact that the polarity of the BOLD response in the inferior parietal lobules reverses from Go trials to No go trials also clearly indicates that brain activity in these areas reflects the type of behavioral response. If BOLD activation reflects a Go response, and BOLD deactivation a No go response than it would be logical to predict BOLD activation during error trials in the No go condition. What can be observed in this study is that MDMA reverses the BOLD response during No go trials in the direction of BOLD activation, suggesting that subjects are more prone to prospective memory errors during intoxication. The latter notion was also supported by a positive correlation between BOLD activation and prospective memory failures in the inferior parietal lobules during No go trials.

In general these data seem to indicate that MDMA suppresses brain processes that are normally involved in prospective memory performance and that the inferior parietal lobules in particular be involved in MDMA induced memory impairment. In addition, brain activations observed during Go trials are likely to be related to performance on the foreground task: ie, monitoring and repeated responding to central stimuli, whereas deactivation of brain activity during No go trials is likely to be related to withholding a response (ie, the prospective task). MDMA acted as a suppressor of BOLD responses observed during placebo treatment, leading to less activation in the Go trial and less deactivation in No go trials. Consequently, the loss of deactivation in inferior parietal lobules may account for the increments in memory failures observed during MDMA intoxication.

\section{DISCLOSURE/CONFLICT OF INTEREST}

The authors declare no conflicts of interest. The authors declare that over the past 3 years they have received funding for research not related to this paper. JR has received grants from pharmaceutical industries, the European Committee, and the Netherlands Organization for Scientific Research (NWO). EF and KK have received grants from NWO.

Trial registration (Nederlands Trialregister)

http://www.trialregister.nl/trialreg/admin/

rctview.asp? $\mathrm{TC}=1416$.

NTR number: NTR1416

Trial name: MDMA and memory

\section{REFERENCES}

Bedi G, Redman J (2008a). Ecstasy use and higher-level cognitive functions: weak effects of ecstasy after control for potential confounds. Psychol Med 38: 1319-1330.

Bedi G, Redman J (2008b). Metamemory in recreational ecstasy polydrug users: what do self-reports of memory failures mean? $J$ Psychopharmacol 22: 872-881.

Bolla KI, McCann UD, Ricaurte GA (1998). Memory impairment in abstinent MDMA ('ecstasy') users. Neurology 51: 1532-1537.

Burgess PW, Quayle A, Frith CD (2001). Brain regions involved in prospective memory as determined by positron emission tomography. Neuropsychologia 39: 545-555.

Burgess PW, Scott SK, Frith CD (2003). The role of the rostral frontal cortex (area 10) in prospective memory: a lateral versus medial dissociation. Neuropsychologia 41: 906-918.

Cole JC, Sumnall HR (2003). The pre-clinical behavioural pharmacology of 3,4-methylenedioxymethamphetamine (MDMA). Neurosci Biobehav Rev 27: 199-217.

Croft RJ, Mackay AJ, Mills AT, Gruzelier JG (2001). The relative contributions of ecstasy and cannabis to cognitive impairment. Psychopharmacology (Berl) 153: 373-379.

Dafters RI, Hoshi R, Talbot AC (2004). Contribution of cannabis and MDMA ('ecstasy') to cognitive changes in long-term polydrug users. Psychopharmacology (Berl) 173: 405-410.

de la Torre R, Farre M, Ortuno J, Mas M, Brenneisen R, Roset PN et al (2000). Non-linear pharmacokinetics of MDMA ('ecstasy') in humans. Br J Clin Pharmacol 49: 104-109.

de la Torre R, Farre M, Roset PN, Pizarro N, Abanades S, Segura M et al (2004). Human pharmacology of MDMA: pharmacokinetics, metabolism, and disposition. Ther Drug Monit 26: 137-144.

den Ouden HE, Frith U, Frith C, Blakemore SJ (2005). Thinking about intentions. Neuroimage 28: 787-796.

Einstein GO, McDaniel MA, Thomas R, Mayfield S, Shank H, Morrisette $\mathrm{N}$ et al (2005). Multiple processes in prospective memory retrieval: factors determining monitoring versus spontaneous retrieval. J Exp Psychol Gen 134: 327-342.

Farre M, de la Torre R, Mathuna BO, Roset PN, Peiro AM, Torrens $M$ et al (2004). Repeated doses administration of MDMA in humans: pharmacological effects and pharmacokinetics. Psychopharmacology (Berl) 173: 364-375.

Forman SD, Cohen JD, Fitzgerald M, Eddy WF, Mintun MA, Noll DC (1995). Improved assessment of significant activation in functional magnetic resonance imaging (fMRI): use of a clustersize threshold. Magn Reson Med 33: 636-647.

Gobel SM, Johansen-Berg H, Behrens T, Rushworth MF (2004). Response-selection-related parietal activation during number comparison. J Cogn Neurosci 16: 1536-1551.

Gouzoulis-Mayfrank E, Daumann J (2006). The confounding problem of polydrug use in recreational ecstasy/MDMA users: a brief overview. J Psychopharmacol 20: 188-193. 
Gouzoulis-Mayfrank E, Daumann J, Sass H (2002). [Chronic neurotoxic damage in ecstasy (MDMA) users. Review of the current state of research]. Nervenarzt 73: 405-421.

Halpern JH, Pope Jr HG, Sherwood AR, Barry S, Hudson JI, Yurgelun-Todd D (2004). Residual neuropsychological effects of illicit 3,4-methylenedioxymethamphetamine (MDMA) in individuals with minimal exposure to other drugs. Drug Alcohol Depend 75: 135-147.

Hanson KL, Luciana M (2004). Neurocognitive function in users of MDMA: the importance of clinically significant patterns of use. Psychol Med 34: 229-246.

Hasler F, Studerus E, Lindner K, Ludewig S, Vollenweider F (2008). Investigation of serotonin-1A receptor function in the human psychopharmacology of MDMA. J Psychopharmacol. Doi: 1177/026g8811080g4650 page 1-13.

Heffernan TM, Jarvis H, Rodgers J, Scholey AB, Ling J (2001a). Prospective memory, everyday cognitive failure and central executive function in recreational users of ecstasy. Hum Psychopharmacol 16: 607-612.

Heffernan TM, Ling J, Scholey AB (2001b). Subjective ratings of prospective memory deficits in MDMA ('ecstasy') users. Hum Psychopharmacol 16: 339-344.

Jager G, de Win MM, van der Tweel I, Schilt T, Kahn RS, van den Brink W et al (2008). Assessment of cognitive brain function in ecstasy users and contributions of other drugs of abuse: results from an FMRI study. Neuropsychopharmacology 33: 247-258.

Kolbrich EA, Goodwin RS, Gorelick DA, Hayes RJ, Stein EA, Huestis MA (2008). Plasma pharmacokinetics of 3,4-methylenedioxymethamphetamine after controlled oral administration to young adults. Ther Drug Monit 30: 320-332.

Kubler A, Dixon V, Garavan H (2006). Automaticity and reestablishment of executive control-an fMRI study. J Cogn Neurosci 18: 1331-1342.

Kuypers KP, Ramaekers JG (2005). Transient memory impairment after acute dose of $75 \mathrm{mg}$ 3.4-methylene-dioxymethamphetamine. J Psychopharmacol 19: 633-639.

Kuypers KP, Ramaekers JG (2007). Acute dose of MDMA (75 mg) impairs spatial memory for location but leaves contextual processing of visuospatial information unaffected. Psychopharmacology (Berl) 189: 557-563.

Kuypers KP, Wingen M, Ramaekers JG (2008). Memory and mood during the night and in the morning after repeated evening doses of MDMA. J Psychopharmacol 22: 895-903.

Lieb R, Schuetz CG, Pfister H, von Sydow K, Wittchen H (2002). Mental disorders in ecstasy users: a prospective-longitudinal investigation. Drug Alcohol Depend 68: 195-207.

Mas M, Farre M, de la Torre R, Roset PN, Ortuno J, Segura J et al (1999). Cardiovascular and neuroendocrine effects and pharmacokinetics of 3,4-methylenedioxymethamphetamine in humans. J Pharmacol Exp Ther 290: 136-145.

McDaniel MA, Einstein GO (1993). The importance of cue familiarity and cue distinctiveness in prospective memory. Memory 1: 23-41.

McGuire P (2000). Long term psychiatric and cognitive effects of MDMA use. Toxicol Lett 112-113: 153-156.

Montgomery C, Fisk JE (2007). Everyday memory deficits in ecstasy-polydrug users. J Psychopharmacol 21: 709-717.

Montgomery C, Fisk JE, Newcombe R (2005). The nature of ecstasy-group related deficits in associative learning. Psychopharmacology (Berl) 180: 141-149.

Morgan MJ (1999). Memory deficits associated with recreational use of 'ecstasy' (MDMA). Psychopharmacology (Berl) 141: $30-36$.
Morgan MJ (2000). Ecstasy (MDMA): a review of its possible persistent psychological effects. Psychopharmacology (Berl) 152: 230-248.

Parrott AC (2000). Human research on MDMA (3,4-methylenedioxymethamphetamine) neurotoxicity: cognitive and behavioural indices of change. Neuropsychobiology 42: 17-24.

Parrott AC (2001). Human psychopharmacology of ecstasy (MDMA): a review of 15 years of empirical research. Hum Psychopharmacol 16: 557-577.

Piazza M, Mechelli A, Butterworth B, Price CJ (2002). Are subitizing and counting implemented as separate or functionally overlapping processes? Neuroimage 15: 435-446.

Pinel P, Le Clec HG, van de Moortele PF, Naccache L, Le Bihan D, Dehaene S (1999). Event-related fMRI analysis of the cerebral circuit for number comparison. Neuroreport 10: 1473-1479.

Poldrack RA, Fletcher PC, Henson RN, Worsley KJ, Brett M, Nichols TE (2008). Guidelines for reporting an fMRI study. Neuroimage 40: 409-414.

Ramaekers JG, Kuypers KP (2006). Acute effects of 3,4-methylenedioxymethamphetamine (MDMA) on behavioral measures of impulsivity: alone and in combination with alcohol. Neuropsychopharmacology 31: 1048-1055.

Rendell PG, Gray TJ, Henry JD, Tolan A (2007). Prospective memory impairment in 'ecstasy' (MDMA) users. Psychopharmacology (Berl) 194: 497-504.

Rodgers J, Buchanan T, Scholey AB, Heffernan TM, Ling J, Parrott A (2001). Differential effects of ecstasy and cannabis on selfreports of memory ability: a web-based study. Hum Psychopharmacol 16: 619-625.

Rodgers J, Buchanan T, Scholey AB, Heffernan TM, Ling J, Parrott AC (2003). Patterns of drug use and the influence of gender on self-reports of memory ability in ecstasy users: a web-based study. J Psychopharmacol 17: 389-396.

Sandrini M, Rossini PM, Miniussi C (2004). The differential involvement of inferior parietal lobule in number comparison: a rTMS study. Neuropsychologia 42: 1902-1909.

Schnell K, Heekeren K, Schnitker R, Daumann J, Weber J, Hesselmann V et al (2007). An fMRI approach to particularize the frontoparietal network for visuomotor action monitoring: detection of incongruence between test subjects' actions and resulting perceptions. Neuroimage 34: 332-341.

Simon NG, Mattick RP (2002). The impact of regular ecstasy use on memory function. Addiction 97: 1523-1529.

Talairach J, Tournoux P (1988). Co-Planar Stereotaxic Atlas of the Human Brain. Thieme Medical: New York.

Thomasius R, Zapletalova P, Petersen K, Buchert R, Andresen B, Wartberg L et al (2006). Mood, cognition and serotonin transporter availability in current and former ecstasy (MDMA) users: the longitudinal perspective. J Psychopharmacol 20: 211-225.

Trimbos I (2007). DIMS jaarbericht 2007: Trimbos Institute (Netherlands Institute of Health and Addiction).

Verkes RJ, Gijsman HJ, Pieters MS, Schoemaker RC, de Visser S, Kuijpers $M$ et al (2001). Cognitive performance and serotonergic function in users of ecstasy. Psychopharmacology (Berl) 153: 196-202.

Zakzanis KK, Campbell Z, Jovanovski D (2007). The neuropsychology of ecstasy (MDMA) use: a quantitative review. Hum Psychopharmacol 22: 427-435.

Zakzanis KK, Young DA, Campbell Z (2003). Prospective memory impairment in abstinent MDMA ('Ecstasy') users. Cogn Neuropsychiatry 8: 141-153. 